\title{
La Mythologie politique de Paul Hadol : des figures du pouvoir à l'épreuve des mythes grecs dans la caricature au début de la Troisième République (1871-1872)
}

Après Cham et Daumier, Hadol, qui esquissait, en bon enfant, sa caricature (je l'ai encore) du bout de la plume sur la rame de papier de la rédaction. Avec son œil brun à fleur de tête, son nez long et épais, sa bouche lippue, elle était, du reste, facile à faire. Mais quelle douceur, quelle innocence dans ce caricaturiste qui, lui, avec des dessins qui ne paraissaient pas toujours, aurait pu fournir plus d'une nouvelle à la main au Véron du Monde illustré ! Le meilleur des hommes, des amis, - ce qui est plus rare, - et je le dis d'après ses amis, au milieu desquels je ne suis que ce qu'on appelle vulgairement une connaissance. (Perreau, 1876, p. 226)

Passant en revue la rédaction du Charivari, le journaliste Adolphe Perreau ${ }^{1}$ évoque avec tendresse le souvenir de Paul Hadol, artiste vosgien né en 1835 qui débute une carrière de dessinateur prolixe dans les années 1860 lorsque le Second Empire commence à se libéraliser. Tout en continuant à produire de nombreuses planches pour La Vie parisienne ou Le Monde illustré, Hadol rejoint la rédaction du Charivari en $1866^{2}$ et lui consacre l'année suivante une superbe lithographie qui met en scène

- Sylvain Nicolle - chercheur associé au Centre d'Histoire Culturelle des Sociétés Contemporaines (CHCSC) de l'Université de Versailles-Saint-Quentin / Paris-Saclay. E-mail : sylvain.nicolle1@orange.fr

ORCID iD : https://orcid.org/0000-0002-7082-1774

1. Il était entré sous le pseudonyme de Paul Girard en décembre 1866 au Charivari, quotidien satirique fondé par Philipon en 1832 et dirigé par Pierre Véron depuis 1865.

2. Sauf omission dans notre dépouillement du Charivari, sa signature n’y apparaît pas avant. 
l'ensemble des collaborateurs du journal exécutant dans un théâtre une œuvre musicale... charivarique ${ }^{3}$ ! S'il n’a jamais atteint la notoriété de ses deux glorieux aînés Cham et Daumier ${ }^{4}$, la postérité a au moins retenu de Hadol la fameuse Carte drolatique d'Europe pour 1870 - jalon incontournable de l'histoire de la cartographie satirique (Barron, 2008) - et La Ménagerie impériale, série de 31 caricatures anti-bonapartistes publiée en 1870 peu après la chute du Second Empire (Tillier, 1995). La censure préventive avait été alors supprimée provisoirement par la Troisième République (Goldstein, 1989) .

Le républicain Hadol poursuit ses incursions dans la caricature politique au cours des mois suivants en passant de la zoologie à la mythologie. Trois jours avant le déclenchement de la Commune à Paris, Le Charivari annonce dans son numéro du 15 mars 1871 qu'il " commencera la semaine prochaine la publication d'une curieuse suite de charges de M. Hadol sous le titre de LA MYTHOLOGIE POLITIQUE ». La première lithographie, "Le Jugement de Thiers-Pâris ", n'est finalement publiée que le 4 avril suivant. Dix autres portraits-charges se succèdent ensuite à intervalle très irrégulier jusqu'au 22 février 1872, "Gambetta-Vulcain " refermant la série 6 . Le fil d'ariane qui lui confère son titre repose sur l'analogie entre une figure du pouvoir des débuts de la Troisième République et une figure de la mythologie grecque, représentée sous la forme d'une divinité (Neptune, Mercure, Jupiter, Vulcain), d'un héros (Sisyphe, Amphion, Achille, Tantale), d’un(e) mortel(le) (Pâris, Pénélope) ou encore d'un monstre (Méduse).

À partir de ce jeu de concordance par lequel Hadol offre une belle preuve visuelle de son esprit de finesse, cet article ambitionne d'éclairer la série des onze portraits-charges selon une double perspective, diachronique et synchronique, au cœur de la recherche sur la caricature du XIX ${ }^{e}$ siècle (Le Men, 2009). On rappellera d'abord en quoi La Mythologie politique s'inscrit dans une tradition iconographique

3. Hadol, «Carte de visite du Charivari », Le Charivari, 2-3 janvier 1867. Le directeur Pierre Véron est à la baguette, qui est surmontée de la marotte, allégorie récurrente du Charivari dans les lithographies qui le font apparaitre sous forme de personnage. Hadol s'est représenté tout en haut des gradins à l'extrémité droite.

4. Henri Maret, qui rédige un «Panthéon de poche des célébrités contemporaines » publié en livraison dans Le Charivari à partir du 15 octobre 1867, consacre à Daumier une brève entrée dithyrambique : «O grand homme, une moitié de la France est à toi, l'autre moitié est à Cham » (Le Charivari, 4 juillet 1868).

5. La censure est officiellement rétablie le 28 décembre 1871 pendant l'état de siège par un décret du gouverneur militaire de Paris. Une circulaire du ministre de l'Intérieur adressée aux préfets étend la mesure à toute la France dès le 11 janvier 1872 (Goldstein, 1989, p. 205).

6. Les onze portraits-charges se trouvent en annexe de cet article avec la date de publication originelle dans Le Charivari. Ils n'ont pas fait l'objet d'une publication spécifique postérieure mais sont conservés ensemble au Musée Carnavalet (QB. 1823 à 1833). Ce recueil informel provient d'un legs de 1911 issu de la collection de caricatures de Maurice Quentin-Bauchart, auteur sous le pseudonyme de Jean Berleux de La Caricature en France pendant la guerre, le siège de Paris et la Commune (18701871) publié en 1890. 
de parodie de l'Antiquité, avant d'analyser successivement les différents critères qui sous-tendent les choix de Hadol : choix biographique des représentés, choix du filtre mythologique qui leur est appliqué, choix formel qui induit une réflexion sur la sémiotique à l'œuvre dans la série. L'épilogue questionnera la portée de celle-ci.

\section{Un thème iconographique hérité : la parodie de l'Antiquité}

La place de l'Antiquité dans la culture du XIX ${ }^{e}$ siècle est contradictoire. Elle est indéniablement centrale en tant que « bloc de représentations qui alimentent un imaginaire social ", mais aussi contestée au nom d'un affranchissement du passé consubstantiel aux idéaux de la Révolution française : la culture antique comme modèle tend à relever désormais du patrimoine (Mélonio, 2008). Or c'est précisément la capacité à accepter ce basculement progressif qui constitue le cœur des polémiques provoquées par des œuvres d'artistes célèbres parodiant l'Antiquité. Les exemples les plus connus concernent la caricature sous la monarchie de Juillet avec la série Histoire ancienne de Daumier (Herding, 1989 ; Wilker, 1996) et les opéras-bouffes d'Offenbach sous le Second Empire, en particulier Orphée aux Enfers en 1854 (Yon, 2010, p. 209212) et La Belle Hélène dix ans plus tard (Yon, 2010, p. 306-308). Cette dimension de la querelle des Anciens et des Modernes au XIX ${ }^{e}$ siècle est en même temps indissociable d'une réflexion sur les usages d'un passé conçu comme une arme critique à l'usage du temps présent.

De ce point de vue, le filtre mythologique est l'un des nombreux procédés explorés bien avant la série de Hadol par la caricature politique. Il est expérimenté dès la fin du XVIII ${ }^{e}$ siècle en Angleterre par l'incontournable James Gillray, d'abord de façon ponctuelle, puis sous la forme de six caricatures regroupées dans une série thématique publiée en mai 1799 sous le titre The New Pantheon of Democratic Mythology L'idée d'une " mythologie politique » est acclimatée en France au début de la monarchie de Juillet dans les planches de La Caricature de Philipon. Elle s'inspire ouvertement du modèle anglais dans le cas de Desperret (Jouve, 1983, p. 47) qui concentre les flèches du journal allégorisé sur l'hydre ministériel (28 mars 1833, pl. n²59). Auparavant, Grandville et Forest avaient parodié l'ensemble du gouvernement dans Les Faux dieux de l'Olympe (20 sept. 1832, pl. $\mathrm{n}^{\circ}$ 200-201), tandis que Louis-Philippe est successivement ridiculisé sous les traits de Cupidon (7 mars 1833, pl. $\mathrm{n}^{\circ} 253$ ), Bacchus (30 janv. 1834, pl. ${ }^{\circ} 355$ ), Hercule ( $1^{\text {er }}$ mai 1834 , pl. $\left.n^{\circ} 382-383\right)$, Ganymède (12 juin 1834, pl. $\mathrm{n}^{\circ}$ 395) ou encore Dédale avec son fils Icare (26 mars 1835, pl. $\mathrm{n}^{\circ}$ 477). La poursuite de cette filiation iconographique sous la Seconde République et le Second Empire, surtout en matière de politique extérieure dans ce dernier

7. La série, répertoriée sous les $n^{\circ} 9374$ à n 9380 dans l'inventaire du British Museum (George, 1942, p. 548-550), s'accompagne d'une notice de présentation pour chaque caricature. On y trouve notamment Fox en Hercule et Walpole en Mars. 
cas pour échapper à la censure, est essentiellement due à Daumier et Cham dans Le Charivari ${ }^{8}$.

Avant que Hadol ne publie dans ce même journal La Mythologie politique, quel rapport entretient-il avec cette tradition parodique de l'Antiquité ? Familier des coulisses de la vie théâtrale, il se passionne pour les opéras-bouffes d'Offenbach, parodiant en image La Belle Hélène une semaine après la première ${ }^{9}$. Il dessine par ailleurs au moins une caricature politique fondée sur la mythologie avant sa série de 18711872. Intitulée "Mars ne sachant à qui donner la bombe " (Le Charivari, 28 juin 1866), Hadol la désigne lui-même comme « le nouveau Jugement de Pâris » devant déterminer si la victoire militaire reviendra à l'Autriche, la Prusse ou son alliée l'Italie dans la guerre austro-prussienne qui s'engage. Une autre caricature présente un statut particulier puisqu'elle est publiée dans Le Charivari parallèlement à La Mythologie politique mais sans faire partie officiellement du cycle : une "hors-série » en quelque sorte. Intitulée "La République selon la droite ou le nouveau cheval de Troie » (23 septembre 1871), elle représente une grande statue de la République qui abrite en son sein le comte de Chambord identifié à Ulysse ${ }^{10}$. Or le prétendant légitimiste réapparaît cinq mois plus tard en Tantale dans la série. Une telle métamorphose interroge donc la temporalité des choix biographiques qu’opère Hadol.

\section{Les choix biographiques : quels critères?}

La notoriété iconographique est un premier indice. En définissant de façon lapidaire la caricature comme " la glace dans laquelle les autres nous voient ${ }^{11}$, Pierre Véron souligne en 1869 son importance comme l'un des vecteurs médiatiques constitutifs de la notion de célébrité (Lilti, 2014). De ce point de vue, les onze personnalités retenues par Hadol bénéficient presque toutes d'un imaginaire caricatural antérieur à sa série mais d'inégale importance ${ }^{12}$. Il est véritablement forgé dès le début

8. En incluant Charles Vernier et Jules Pelcoq, nous avons relevé près de trente exemples entre 1848 et 1870. La fondation de Punch or The London Charivari en 1843 facilite par ailleurs le processus de transfert culturel entre l'Angleterre et la France. Voir par exemple l'élection présidentielle du 10 décembre 1848 traitée à travers le filtre du Jugement de Pâris dans Punch (28 octobre 1848) et Le Charivari (18 novembre 1848).

9. Hadol, «Théâtre des Variétés - La Belle Hélène. Enlèvement-Bouffe en trois actes ", La Vie parisienne, 24 décembre 1864.

10. Le même motif avait déjà été exploité sous l'Empire libéral deux mois après la formation du ministère Emile Olliver : Thiers-Ulysse était caché à l'intérieur d'un véritable cheval (incarnant le parlementarisme) tiré par les députés libéraux en direction du Corps législatif. Voir Stop (Louis Morel-Retz, dit), « Le Cheval de Troie », Le Charivari, 4 mars 1870.

11. Fantasio (pseudonyme de Pierre Véron), « Dictionnaire de l'avenir », Le Charivari, 10 octobre 1869.

12. Nous n'avons pas retrouvé de caricatures de Léon Say et de l'amiral Pothuau avant Hadol mais une enquête très approfondie pourrait peut-être infirmer cette conclusion provisoire. 
de la monarchie de Juillet pour Thiers ${ }^{13}$, mais seulement esquissé sous la Deuxième République à l'égard du comte de Chambord ${ }^{14}$ et de Rouher ${ }^{15}$. Cet imaginaire caricatural est bien plus récent pour tous les autres. Pouyer-Quertier, Gambetta et Grévy sont croqués à la fin du Second Empire par Gill dans L'Eclipse ${ }^{16}$, tandis que les portraits-charges de Trochu, Faidherbe et Littré côtoient ceux de Rouher, Thiers et Gambetta dans les séries incontournables publiées au début de la Troisième République : Le Pilori-Phrénologie de Belloguet (novembre-décembre 1870) ${ }^{17}$; Fruits, fleurs et légumes du jour d'Alfred Le Petit (janvier-mars 1871) ${ }^{18}$; Le Musée-homme ou le jardin des bêtes de Faustin (1871) ${ }^{19}$. En produisant La Mythologie politique, Hadol puise dans le fond biographique commun à ces trois séries tout en délaissant d'autres " célébrités politiques " contemporaines incontournables, en particulier les « trois Jules» : Favre, Ferry et Simon.

La fonction politique occupée au moment de la publication du portrait-charge peut se diviser en trois catégories ${ }^{20}$. Ceux qui exercent le pouvoir exécutif : Thiers est chef du pouvoir exécutif de la République française (décret du 17 février 1871), l’amiral Pothuau et Pouyer-Quertier font partie du gouvernement - le premier comme ministre de la Marine et des Colonies (19 février 1871), le second comme ministre des Finances (25 février 1871) -, Léon Say est préfet de la Seine (6 juin 1871). Ceux qui sont représentants du peuple : Grévy préside l'Assemblée nationale où siègent également Trochu (au centre droit), Littré (à gauche) et Gambetta (à l'extrême-gauche). Ceux enfin qui n'exercent pas (ou plus) de fonction de politique : Rouher se présente aux législatives du 2 juillet 1871 pour rejoindre l'Assemblée Nationale, Faidherbe en a démissionné le 26 août $1871^{21}$ et le comte de Chambord fait figure d'éternel prétendant à la couronne.

13. Voir la charge de Daumier dans Le Charivari, 2 juin 1833. Thiers figure aussi en bonne place dans $\mathrm{La}$ Caricature de Philipon avant que les lois de septembre 1835 ne rétablissent la censure préventive. Il fut certainement l'homme de pouvoir le plus caricaturé du XIX ${ }^{e}$ siècle, ce qui s'explique par sa longévité politique exceptionnelle.

14. Voir les lithographies de Charles Vernier lorsque la question de la fusion est - déjà ! - à l'ordre du jour (Le Charivari, 27 mai et 27 septembre 1851).

15. Daumier lui consacre le $\mathrm{n}^{\circ} 30$ de sa série « Les représentans représentés » (2e partie, Assemblée législative) et le représente aussi avec de Parrieu (Le Charivari, 3 septembre 1850).

16. Voir le $\mathrm{n}^{\circ}$ 22, 21 juin 1868 ( «M. Pouyer-Quertier») et le $\mathrm{n}^{\circ}$ 44, 22 novembre 1868 («Quelques avocats ». Les républicains du barreau de Paris sont représentés par Arago, Gambetta, Grévy, Laurier et Crémieux).

17. Rouher $\left(n^{\circ} 7\right)$, Trochu ( $\left.n^{\circ} 10\right)$, Thiers ( $\left.n^{\circ} 11\right)$. Voir Tillier (1997, p. 140).

18. Trochu $\left(n^{\circ} 1\right)$, Gambetta $\left(n^{\circ} 2\right)$, Faidherbe ( $\left.n^{\circ} 7\right)$, Littré $\left(n^{\circ} 11\right)$, Thiers ( $\left.n^{\circ} 17\right)$, Pouyer-Quertier (n²2), Grévy ( $\left.n^{\circ} 24\right)$. Voir Tillier (1997, p. 133).

19. Trochu ( $\left.{ }^{\circ} 1\right)$, Thiers ( $\left.n^{\circ} 2\right)$, Grévy ( $\left.{ }^{\circ} 13\right)$. Voir Tiller (1997, p. 137).

20. Pour une approche biographique générale, nous renvoyons aux notices des dictionnaires de Robert, Bourloton et Cougny (1889-1891), Vapereau (1893) et Yvert (1990).

21. Il protestait contre le vote des pouvoirs constituants à l'Assemblée d'où le thème de la retraite d'Achille. La lecture de sa lettre de démission, dont les termes sont jugés blessants par la droite, suscite un léger incident. Voir Journal officiel de la République française, 27 août 1871, p. 3011. 
L'ordre d'apparition des onze portraits-charges est déterminé par leur date de publication qui s'étire sur dix mois dans Le Charivari. L'effet d'opportunité se présente avec une acuité particulière à deux reprises. Anticipation d'un résultat espéré : la caricature du bonapartiste Rouher est publiée une semaine avant son double échec aux législatives partielles du 2 juillet 1871. Commentaire rapproché de l'événement : la caricature de Littré est publiée onze jours après son élection à l'Académie-Française le 30 décembre 1871 qui a entraîné la bruyante démission - finalement ajournée de Mgr Dupanloup. En revanche, le lien avec l'actualité brûlante est moins marqué pour d'autres portraits-charges, et peut même apparaître assez déphasé dans le cas de Gambetta qui clôt la série. Au-delà des circonstances propres à la publication de chaque caricature, un regard panoramique sur la série invite à interroger l'existence d'une cohérence thématique à travers le choix des filtres mythologiques.

\section{Le choix des filtres mythologiques : une cohérence thématique?}

Trois caricatures relèvent du cycle de Troie. Le Jugement de Thiers-Pâris restitue d'emblée le champ des possibles dans le choix du régime politique de la France, à l'exception d'un retour à l'Empire dont Hadol ne veut surtout pas envisager l'hypothèse. Le cycle troyen est renoué avec les portraits-charges de Pénélope-Trochu et AchilleFaidherbe qui font écho à deux tempéraments militaires opposés dans un contexte de défaite : Trochu - nommé le 4 septembre 1870 président du Gouvernement de la Défense nationale - ou l'art de gagner du temps qui tourne au défaitisme par excès de prudence ; Faidherbe - nommé commandant en chef de l'armée du Nord par Gambetta le 3 décembre 1870 - ou l'art de résister courageusement face à l'ennemi pour retarder la défaite.

Gambetta, précisément, est l'une des trois divinités olympiennes dont le rôle rappelle dans la série l'importance du verbe politique. Verbe maîtrisé qui s'incarne dans un discours performatif : l'homme fort de la Délégation de Tours qui cumule les ministères de l'Intérieur et de la Guerre forge littéralement, avec l'aide de deux cyclopes $^{22}$, les décrets et proclamations qui en font le grand artisan de la résistance patriotique (Mayeur, 2008, p. 92-132). Verbe au contraire échappé devant une assemblée qui est l'émanation de la souveraineté nationale : la médiocrité oratoire de l’amiral Pothuau l'empêche d'ancrer une véritable parole souveraine qui rend la comparaison à Neptune dérisoire ; tout aussi dérisoire paraît la colère de Jupiter-Grévy qui tonne depuis son perchoir par la voix et par le geste - la foudre jaillit de la sonnette

22. L'identification n'est pas absolument certaine mais il s'agit sans doute au premier plan de Clément Laurier (directeur du personnel au ministère de l'Intérieur) et à l'arrière-plan de Frédéric Steenackers (directeur général des Postes et des Télégraphes). Le choix des cyclopes s'explique par le lieu souterrain mais leur visage de borgne renvoie aussi à l’oil énucléé de Gambetta. 
du président de l'Assemblée Nationale - sans parvenir à dompter un auditoire survol$t^{2} e^{23}$. La quatrième divinité olympienne individualisée, Mercure, déplace subrepticement le registre souverain de la parole à l'argent. Pouyer-Quertier maitrisait en effet autant la première - il passait pour un maître Jacques - qu'il savait manier le second : après avoir participé aux négociations sur l'indemnité de guerre due à l'Allemagne ${ }^{24}$, le ministre des Finances fut chargé de préparer l'emprunt destiné à la payer et s'en acquitta avec succès tout en préparant une liste interminable de nouveaux impôts.

Les héros, qui ne relèvent pas du cycle troyen à l'exception d'Achille, peuvent se distinguer en deux catégories. D'un côté, les héros maudits, victimes de la némésis, qui sont confrontés à leur impuissance politique : Sisyphe-Rouher est condamné à soulever perpétuellement le rocher du bonapartisme qui s'apparente à un véritable boulet politique, ce qui rend ainsi illusoire pour le républicain Hadol tout espoir de restauration impériale par les urnes ; Tantale-Chambord, viscéralement attaché au droit divin et au drapeau blanc, ne peut assouvir son désir d'exercer le pouvoir monarchique qu'il a pourtant littéralement à portée de main, et sa métamorphose qui le fait passer en cinq mois du rusé Ulysse au supplicié Tantale (supra, \$1) ne saurait mieux résumer en image l'échec annoncé du chambordisme (De Waresquiel, 2015). À la fatalité s'oppose le volontarisme : tel Amphion qui bâtit les murailles de Thèbes au son de sa lyre, Léon Say lance la reconstruction de Paris après la Commune en faisant exécuter les travaux de voiries les plus urgents. Le langage musical du prodige repose évidemment sur une réalité prosaïque : si le héros antique faisait payer son spectacle en faisant travailler directement les spectateurs à l'édification des remparts ${ }^{25}$, le préfet de la Seine fait financer ses travaux par les contribuables grâce à une partie de l'emprunt de 350 millions demandé par la ville de Paris et autorisé par l'Assemblée Nationale ${ }^{26}$.

Le seul monstre individualisé de la série, Méduse, dont la tête animée surgit de l'égide d'Athéna, met en scène de façon spectaculaire la capacité à terrasser ses ennemis sur le plan intellectuel. Son pouvoir de pétrification fait de Littré le chantre du positivisme qui fait triompher le progrès de l'esprit humain face à l'obscurantisme clérical incarné par Mgr Dupanloup et les académiciens qui le soutiennent ${ }^{27}$. Le choix de Méduse rappelle aussi au premier degré la laideur proverbiale du célèbre lexicographe - « quelle singulière idée a eue la nature de mettre une lettre si intéressante

23. À rapprocher de la série de Faustin (voir supra) : Grévy est représenté comme Président du Palais des Singes.

24. Elle s'élève à cinq milliards de francs, somme fixée dans le traité préliminaire de paix signé le 26 février 1871 (art. 2) et confirmée dans le traité de Francfort du 10 mai suivant (art. 7).

25. Voir la version du mythe dans Palaiphatos, Histoires incroyables, chap. 41 (Zéthos et Amphion).

26. Voir Journal officiel de la République française, 7 septembre 1871, p. 3260-3262.

27. Dupanloup s’était opposé avec succès à l'élection de Littré à l'Académie française en 1863 et avait rédigé à cette occasion le pamphlet Avertissement à la jeunesse et aux pères de famille sur les attaques dirigées contre la religion par quelques écrivains d'aujourd'hui (1863). 
dans une si vilaine enveloppe !» (Véron, 1875, p. 186) - et invite à analyser désormais les choix formels de Hadol dans sa série.

\section{Les choix formels : quel fonctionnement sémiotique?}

Les portraits-charges de La Mythologie politique s'inscrivent d'un point de vue formel dans la tradition ouverte par Le Panthéon Charivarique de Benjamin Roubaud (Guillaume et Le Men, 1988). Cette série de 99 lithographies, initialement publiées dans Le Charivari entre 1838 et 1841, proposait une galerie de célébrités du monde littéraire, théâtral, musical et artistique dont la représentation reposait systématiquement sur le contraste entre un corps atrophié et une tête hypertrophiée, principe dit de la «grosse tête » hérité du grotesque (Baridon et Guédron, 2015). S’y ajoutait une légende-texte, qui fut formalisée en quatrain dans la série Nouveau Panthéon Charivarique, publiée une génération plus tard dans le même journal ${ }^{28}$. Paul Hadol en fut précisément le fer de lance puisqu'il réalisa 19 des 25 portraits-charges alors qu'il venait juste de rejoindre la rédaction du Charivari, et ce coup d'éclat contribua sans doute à asseoir sa réputation de caricaturiste si l'on en croit Henry Maret ${ }^{29}$. En somme, Hadol avait déjà expérimenté et « rôdé » le dispositif sémiotique grosse-tête / légende versifiée qu'il réinterprétait dans La Mythologie politique.

Le filtre mythologique implique la représentation des attributs usuels permettant d'identifier les personnages et se double parfois d'un jeu d'écriture interne à l'image : la scène mythologique devient un rébus politique. Hadol utilise par exemple la vaste superficie offerte par le rocher poli de Sisyphe-Rouher afin d'y inscrire les tares associées au bonapartisme selon un principe de régression chronologique : l'humiliation patriotique lors de la défaite finale en 1870 (Sedan, Metz) et outre-Atlantique en 1867 (Mexique) ; le viol de la légalité lors du coup d'État réussi en 1851 (2 décembre) après les tentatives ratées de 1836 et 1840 (Strasbourg, Boulogne). Le même procédé se retrouve miniaturisé dans Le Jugement de Thiers-Pâris et Tantale-Chambord : l'inscription sur les fruits produit un effet métonymique qui gomme toute ambiguité sur la lecture politique induite par la scène mythologique représentée. Le rébus fonctionne à l'identique avec les autres symboles. Sur le portrait-charge de GambettaVulcain, le ballon dessiné sur le mur fait référence à L'Armand-Barbès qui avait permis à Gambetta de quitter le 7 octobre 1870 Paris assiégé, tandis que l'inscription « chassepots » dans la partie inférieure renvoie à la fabrication des fusils à aiguilles

28. Sa publication s'étire du 9 mars 1866 au 6 avril 1867. Cette série et les suivantes seront analysées dans un article que nous préparons sur les différents Panthéons du Charivari au cours de la seconde moitié du XIX ${ }^{e}$ siècle.

29. Ce dernier lui consacre trois quatrains dans son « Panthéon de poche des célébrités contemporaines » déjà cité. Le troisième énonce la supériorité du caricaturiste sur le publiciste : «Mon Panthéon n'est pas solide / Ses nombreux héros crouleront / Le tien ne sera jamais vide / Et tes images resteront. » Voir Le Charivari, 17 avril 1869. 
adoptés par l'armée française depuis 1866. Quant au foudre jupitérien visible derrière... Vulcain, il désigne par une double métonymie Gambetta comme un foudre de guerre et un foudre d'éloquence. Hadol se cite subtilement dans ce dernier cas : il avait déjà représenté en 1869 Gambetta en député aiguisant un foudre sur une meule pour suggérer les séances orageuses que la parole tonnante de l'irréductible opposant républicain pouvait provoquer en faisant monter très haut la température dans l'enceinte du Corps législatif ${ }^{30}$ !

Les subtiles citations artistiques, littéraires, voire musicales, jouent ainsi sur la connivence que Hadol espère nouer avec son public. La figure de la République, à la fois virile et dénudée dans le Jugement de Thiers-Pâris, s'inspire visiblement de l'esquisse que Daumier avait réalisée en 1848 afin de magnifier l'idéal d'une République forte et nourricière (Agulhon, 2001). De la peinture, Hadol glisse à la sculpture. L'amiral Pothuau s'identifie à Neptune par son trident mais les chevaux marins qui jaillissent du verre d'eau du ministre-orateur se réfèrent autant à ceux d'Apollon sur son char, groupe sculpté de Tuby (1668-1670) émergeant du bassin d'Apollon que les parlementaires peuvent admirer dans les jardins de Versailles au cœur de la grande perspective $^{31}$. À ces formes d'intericonicité s'ajoute un jeu de pastiche littéraire. Les fables de La Fontaine s'invitent malicieusement dans deux portraits-charges. Le chœur charmant des grenouilles chantant la sérénade à Tantale-Chambord sur les bords du Styx demande désespérément un roi, tandis que le riant batracien prussien qui avale les pièces d'or lancées par Mercure-Pouyer-Quertier pour le nourrir a tout l'air d'une grenouille qui veut se faire plus grosse que le bœuf. Enfin, on peut prêter l'oreille à un discret fond musical. La représentation d'Achille-Faidherbe attablé devant son café et son journal peut renvoyer au goût de l'anachronisme truculent de Meilhac et Halévy dans le livret de La Belle Hélène. Quant à Pénélope-Trochu, qui délaisse sa pelote de laine pour rouler et dérouler son plan censé être le deus ex-machina du siège de Paris, Hadol ne compte-t-il pas sur le lecteur du Charivari pour lui faire entonner ce qui est devenu une arlésienne militaire à succès au cours des semaines qui précèdent sa caricature ${ }^{32}$ ?

30. Hadol, «Le Thermomètre parlementaire », Le Charivari, 13 décembre 1869. Bancel, Raspail et Rochefort se situent comme Gambetta au-dessus de la barre des $40^{\circ} \mathrm{C}$ !

31. Rappelons que l'Assemblée Nationale siège à l'Opéra-Royal depuis le 20 mars 1871.

32. Voir Le Plan de Trochu. Seule histoire vraie du siège de Paris. La chanson, illustrée d'une lithographie en couleur de Lefman et Lourdel, est vendue 25 centimes. Le Grelot en explique la genèse $\left(\mathrm{n}^{\circ} 19,20\right.$ août 1871), se plaint de sa saisie provisoire par les agents de la préfecture de police $\left(\mathrm{n}^{\circ} 21,3\right.$ septembre $1871)$ et précise qu'après l'épuisement du $5^{\mathrm{e}}$ tirage, une édition de luxe sera mise en vente à 1 franc $\left(\mathrm{n}^{\circ}\right.$ 27, 15 octobre 1871). La caricature de Hadol est publiée le 9 octobre. 


\section{Épilogue : la portée de La Mythologie politique}

La série ne fut pas un chant du cygne puisqu'elle exerça une incontestable influence sur d'autres caricaturistes. Gill cite fidèlement Hadol dans L'Eclipse en chargeant à nouveau Rouher en Sisyphe ( $\mathrm{n}^{\circ} 188,2$ juin 1872), avant d'adapter les filtres mythologiques : il fait d'abord de Thiers un Tantale soumis au supplice du verre d'eau de l'orateur ( ${ }^{\circ} 223,2$ février 1873), puis un nouvel Achille appâtant Cerbère - les trois droites - sous l'œil menaçant d'un Gambetta probable myrmidon ( ${ }^{\circ} 224,9$ février 1873). D’autres exemples postérieurs pourraient être avancés et incitent à penser que le procédé se prolongea au moins jusqu'au début du $\mathrm{XX}^{\mathrm{e}}$ siècle ${ }^{33}$.

L’analogie a-t-elle véritablement trouvé son public ? En commentant lui-même dans Le Don Quichotte sa caricature hebdomadaire qui représentait sur le corps de Cerbère les têtes de trois députés radicaux - Clemenceau, Madier-Montjau et Lockroy ( ${ }^{\circ} 257,23$ mai 1879), Charles Gilbert-Martin regrettait dans un lamento classique le scepticisme des Modernes induit par la référence mythologique, perçue comme anachronique, tout en soulignant la déperdition intellectuelle qui pouvait en résulter :

Nous n'avons plus de déférence,

Pour les Dieux qu'Homère a chantés ;

Mais, plus souvent que l'on ne pense,

Les fables sont des vérités.

Pour moi dans la mythologie

Je vois de très grandes leçons,

Et jéprouve la nostalgie

De tous ces Dieux si bons garçons.

Effritement du soubassement mythologique de la culture antique alors même qu'il offre toujours la possibilité d'un précieux détour intellectuel pour mettre en perspective un présent ne pouvant se suffire à lui-même. À l'heure où le présentisme poursuit précisément son œuvre de table rase, parfois jusqu'à l'hybris, il y a là sans doute une leçon intemporelle à méditer...

33. Notre enquête en cours nous a déjà conduit à retrouver une quinzaine de caricatures dans la presse satirique française entre 1872 et 1900 . À l'échelle internationale, voir par exemple le cas des États-Unis avec Théodore Roosevelt assimilé à Hercule («The Infant Hercules and the Standard Oil serpents », Puck, 23 mai 1906). 


\section{RÉFÉRENCES}

Agulhon, M. (2001) [1979]. Marianne au combat. L'imagerie et la symbolique républicaines de 1789 à 1880. Paris : Flammarion.

Baridon, L. et Guédron, M. (2015). L'Art et l'histoire de la caricature. Paris : Citadelles \& Mazenod. Barron, Roderick. M. (2008). Bringing the map to life: European satirical maps, 1845-1945. Belgeo, 3-4, 445-464. https://journals.openedition.org/belgeo/11935

De Waresquiel, E. (dir.). (2015). Le Lys et la République. Henri, comte de Chambord. 18201883. Paris : Tallandier.

George, M. D. (1942). Catalogue of Political and Personal Satires Preserved in the Department of Prints and Drawings in the British Museum, vol. VII, 1793-1800. London : British Museum.

Goldstein, R. (1989). Censorship of Political Caricature in Nineteenth-Century France. London : Kent State University Press.

Guillaume, V. et Le Men, S. (1988). Benjamin Roubaud et le Panthéon charivarique. Paris : Maison de Balzac.

Herding, K. (1989). Daumier critique des temps modernes. Recherches sur l'histoire ancienne. Gazette des Beaux-Arts, 6/113, 29-44.

Jouve, M. (1983). L'âge d'or de la caricature anglaise. Paris : Presses de la Fondation Nationale des Sciences Politiques.

Le Men, S. (2009). La recherche sur la caricature du XIX siècle : état des lieux. Perspective, 3, 426-460. https://journals.openedition.org/perspective/1332

Lilti, A. (2014). Figures publiques. Naissance de la célébrité, 1750-1850. Paris : Fayard.

Mayeur, J.-M. (2008). Léon Gambetta. La Patrie et la République. Paris : Fayard.

Mélonio, F. (2008). L'Antiquité au temps de Daumier et Tocqueville : qui nous délivrera des Grecs et des Romains ? Bulletin de l'Association Guillaume Budé, 1, 64-85. https:// www.persee.fr/doc/bude_0004-5527_2008_num_1_1_2278

Perreau, A. (dit Maxime Rude). (1876). Confidences d’un journaliste. Paris : Librairie André Sagnier.

Robert, A., Bourloton, E. et Cougny, G. (dir.). (1889-1891). Dictionnaire des parlementaires français [...], 5 vol. Paris : Bourloton éditeur. http://www2.assemblee-nationale.fr/ sycomore/recherche

Tillier, B. (1995). Paul Hadol et sa ménagerie ! ou le cirque d'un caricaturiste anti-bonapartiste. Gavroche, $80,1-8$.

Tillier, B. (1997). La RépubliCature. La caricature politique en France. 1870-1914. Paris : CNRS éditions.

Vapereau, G. (dir.). (1893). Dictionnaire universel des contemporains [...], $6^{\text {ème }}$ éd. Paris : Hachette.

Véron, P. (1875). « Littré ». Dans Le Panthéon de poche. Variations sur mes contemporains. Paris : Decorce-Cadot.

Wilker, J.-S. (1996). Daumier's Histoire ancienne : French Classical Parody in the 1840s. New York University: Graduate schools of Arts and Science.

Yon, J.-C. (2010). Jacques Offenbach. Paris : Gallimard.

Yvert, B. (dir.). (1990). Dictionnaire des ministres (1789-1989). Paris : Perrin. 


\section{FIGURES}

Hadol, P. (4 avril 1871). Le Jugement de Thiers-Pâris. Le Charivari. BnF.

https://gallica.bnf.fr/ark:/12148/bpt6k3068148w/f3.item

Hadol, P. (26 juin 1871). Sisyphe-Rouher. Le Charivari. BnF.

https://gallica.bnf.fr/ark:/12148/bpt6k3068179f/f3.item

Hadol, P. (10 juillet 1871). Neptune (amiral Pothuau). Le Charivari. BnF.

https:/gallica.bnf.fr/ark:/12148/bpt6k3068193q/f3.item

Hadol, P. (29 juillet 1871). Mercure (Pouyer-Quertier). Le Charivari. BnF.

https:/gallica.bnf.fr/ark:/12148/bpt6k3068212m/f3.item

Hadol, P. (11 août 1871). Amphion-Léon Say. Le Charivari. BnF.

https://gallica.bnf.fr/ark:/12148/bpt6k30682257/f3.item

Hadol, P. (31 août 1871). Jupiter-Grévy. Le Charivari. BnF.

https://gallica.bnf.fr/ark:/12148/bpt6k3068244k/f3.item

Hadol, P. (9 octobre 1871). Pénélope-Trochu. Le Charivari. BnF.

https://gallica.bnf.fr/ark:/12148/bpt6k3068283p/f3.item

Hadol, P. (24 nov. 1871). Le général Faidherbe (Achille). Le Charivari. BnF.

https://gallica.bnf.fr/ark:/12148/bpt6k3068328t/f3.item

Hadol, P. (10 janvier 1872). La tête de Méduse-Littré. Le Charivari. BnF.

https:/gallica.bnf.fr/ark:/12148/bpt6k3063681p/f3.item

Hadol, P. (22 février 1872). Tantale-de-Chambord. Le Charivari. BnF.

https:/gallica.bnf.fr/ark:/12148/bpt6k3063723k/f3.item

Hadol, P. (27 février 1872). Gambetta-Vulcain. Le Charivari. BnF.

https:/gallica.bnf.fr/ark:/12148/bpt6k3063728n/f3.item

RÉSUMÉ : Entre 1871 et 1872, le dessinateur Paul Hadol publie dans l'hebdomadaire satirique Le Charivari onze portraits-charges formant une série intitulée La Mythologie politique. Cet article rappelle d'abord comment celle-ci s'inscrit dans une tradition préexistante de parodie iconographique de l'Antiquité qui naît en Angleterre à la fin du XVIII ${ }^{e}$ siècle avant d'être acclimatée en France à partir de la monarchie de Juillet. On interroge ensuite les critères de sélection biographique des célébrités caricaturées et la cohérence thématique possible de la série à partir de la typologie des filtres mythologiques - le cycle de Troie, les divinités olympiennes, les héros, les monstres. Enfin, l'analyse plus spécifiquement sémiotique des caricatures met en évidence la reprise du procédé associant grosse-tête et légende versifiée aux deux séries du Panthéon charivarique (1838-1841 et 1866-1867), la dimension de rébus politique, et le jeu de citations artistiques, littéraires voire musicales. L'épilogue montre que la portée de la série peut s'envisager d'un point de vue politique et artistique mais aussi intellectuel dans le rapport au temps qu'elle questionne.

Mots-clés : caricature politique, mythologie, Antiquité, Paul Hadol, Le Charivari 
"The Political Mythology" of Paul Hadol: figures of power as Greek mythological characters in caricature at the beginning of the Third Republic (1871-1872)

ABSTRACT: Between 1871 and 1872, the cartoonist Paul Hadol published in the satirical weekly Le Charivari, eleven loaded portraits forming a series entitled La Mythologie politique. This article first places the series against the backdrop of the pre-existing tradition of iconographic parody of antiquity that began in England at the end of the 18th century, before being adopted in France during the July Monarchy. We then question the biographical criteria for the selection of the caricatured celebrities, and the potential thematic coherence of the series based on the typology of mythological filters - the cycle of Troy, the Olympian deities, heroes, monsters. Finally, the more specifically semiotic analysis of the caricatures highlights the resumption of the process associating big-head and versified legend with the two series of the Charivaric Pantheon (1838-1841 and 1866-1867), the dimension of political rebus, and the game of artistic, literary and even musical quotes. The conclusion shows that the scope of the series can be considered from a political and artistic point of view, but also intellectually in relation to the time which it concerns.

Keywords: political caricature, mythology, antiquity, Paul Hadol, Le Charivari 


\section{Annexe : Les 11 portraits-charges de La Mythologie politique publiés dans Le Charivari. BnF.}

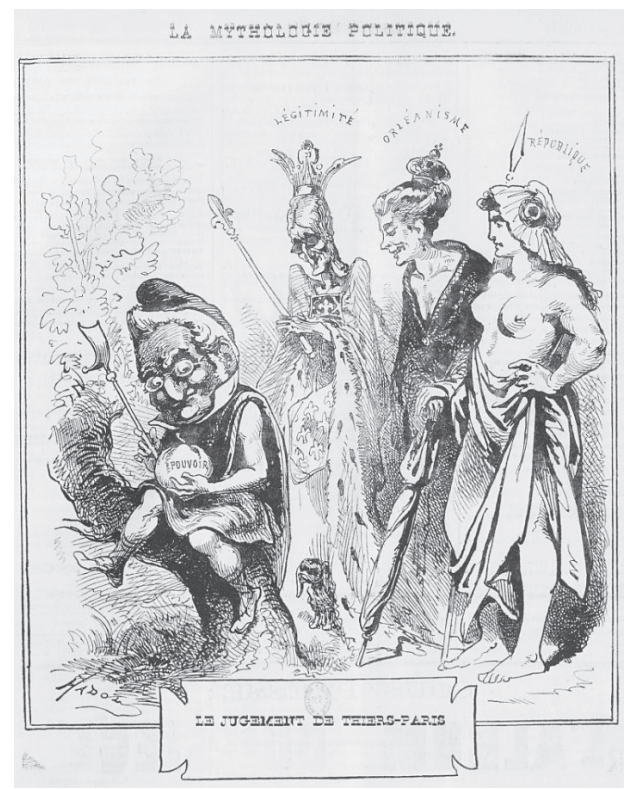

4 avril 1871 : Le Jugement de Thiers-Pâris

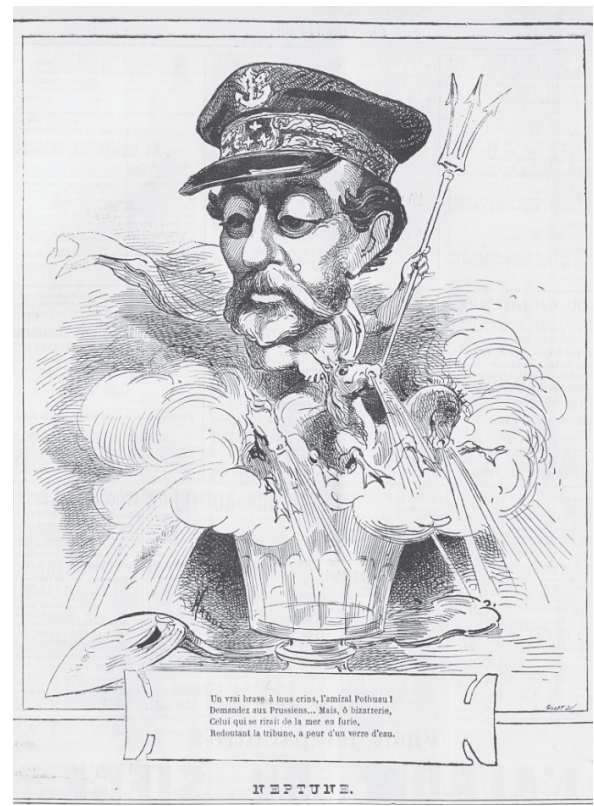

10 juillet 1871 : Neptune (amiral Pothuau)

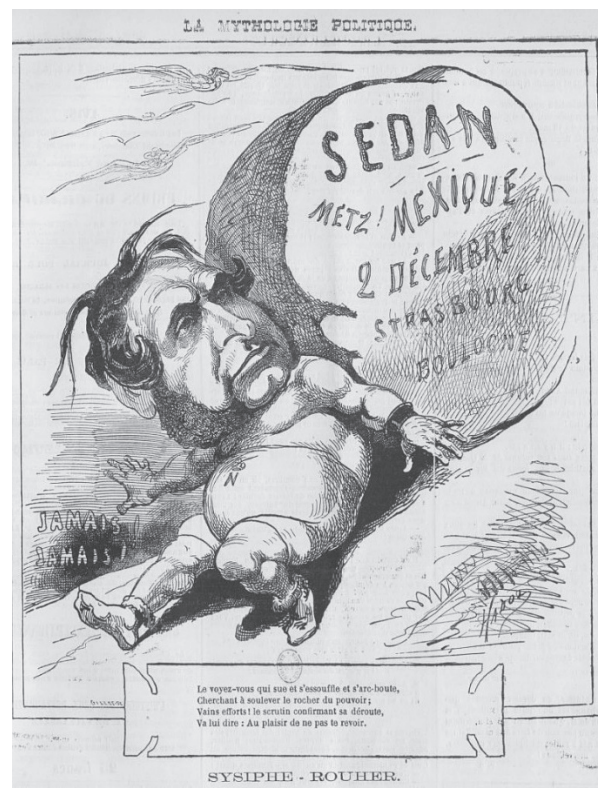

26 juin 1871 : Sisyphe-Rouher

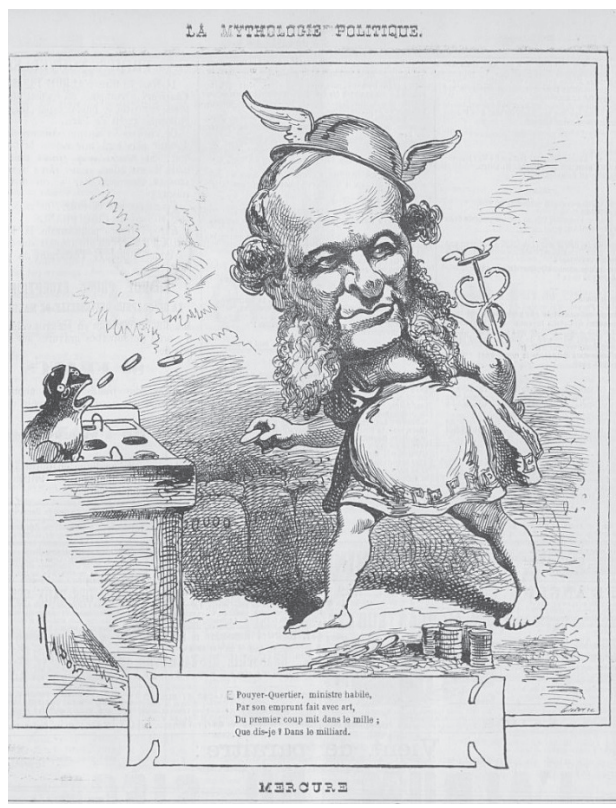

29 juillet 1871 : Mercure (Pouyer-Quertier) 


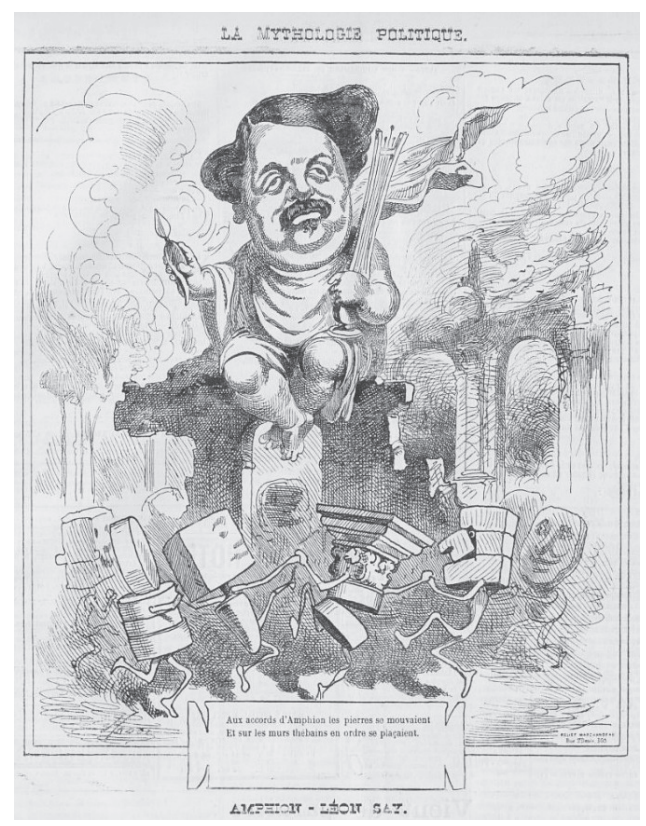

11 août 1871 : Amphion-Léon Say

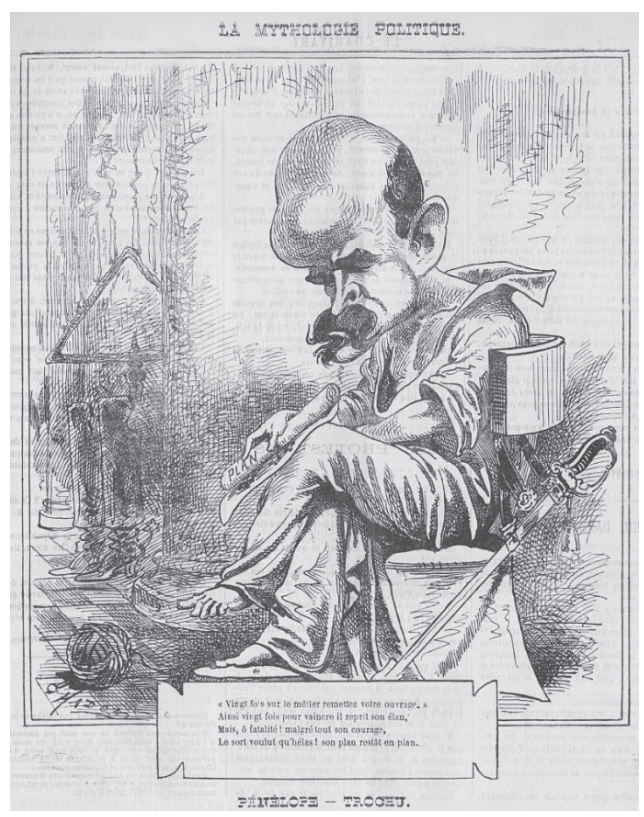

9 octobre 1871 : Pénélope-Trochu

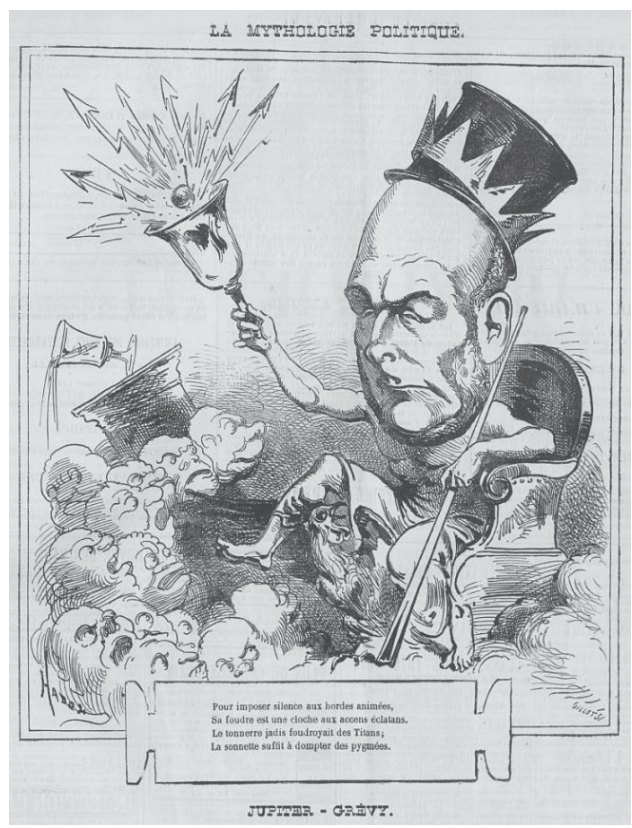

31 août 1871 : Jupiter-Grévy

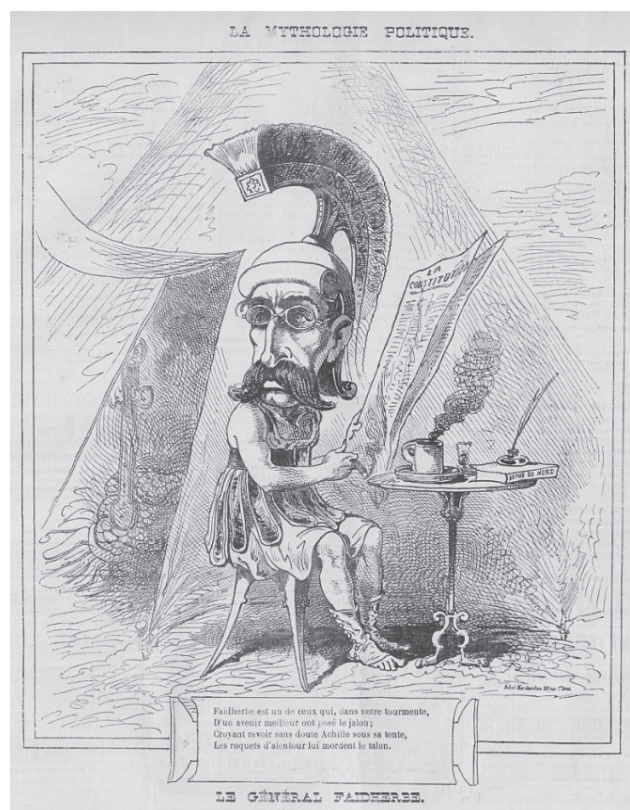

4 nov. 1871 : le général Faidherbe (Achille) 


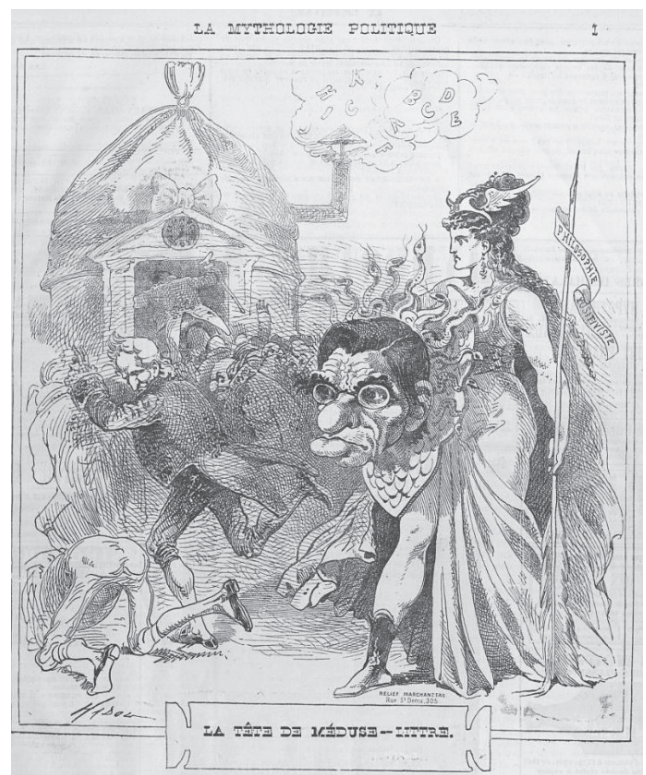

10 janvier 1872 : la tête de Méduse-Littré

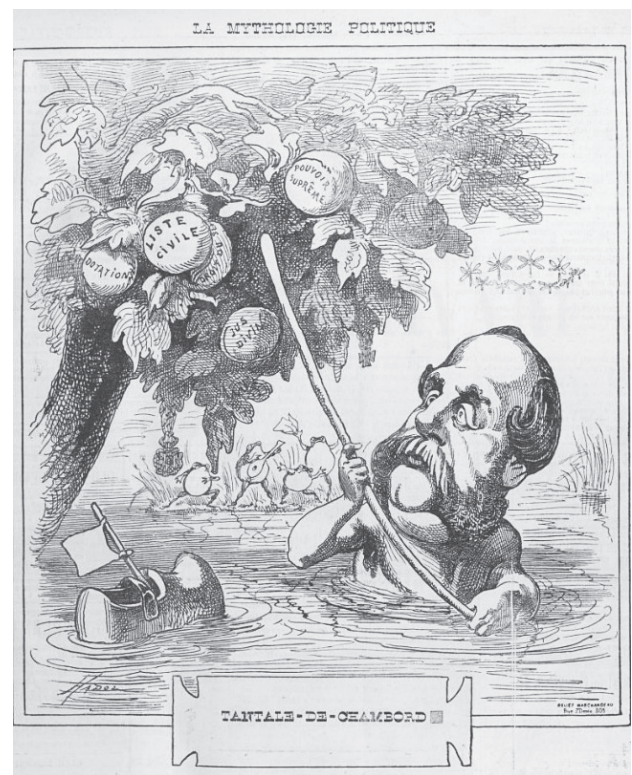

22 février 1872 : Tantale-de-Chambord

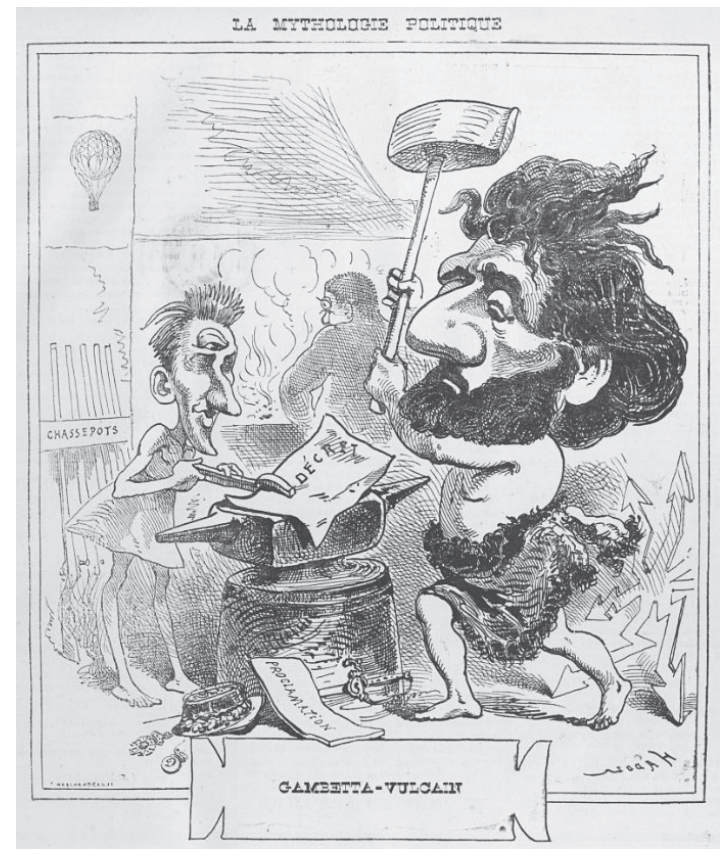

27 février 1872 : Gambetta-Vulcain 